\title{
A clinical algorithm for the management of facial nerve palsy from an oculoplastic perspective
}

\begin{abstract}
Background/Aims Facial nerve palsy can be a sight-threatening complication. We have developed a flow diagram to aid in the management of these patients so that corneal complications may be avoided. This involves the recognition of a facial palsy and institution of treatment as guided by the flow chart. Method Fifty-six patients suffered a facial nerve palsy following acoustic neuroma surgery. All received regular topical ocular lubrication, followed by either botulinum toxin A (BTXA)-induced ptosis (if corneal exposure developed despite conservative treatment) or definitive eyelid surgery.

Results Twenty-one patients required regular lubrication only. Of these patients treated for comeal exposure, 20 received BTXA with good resulting corneal cover. Unfortunately, 9 of these suffered diplopia, although in 4 this resolved quickly. Twenty-four patients underwent a total of 64 eyelid procedures including levator recession, lateral tarsorraphy, lateral canthal sling, medial canthoplasty and gold weight insertion. All patients had good corneal cover post-operatively and were cosmetically improved. Of the 56 patients with a facial nerve palsy, 7 presented with a corneal epithelial defect or an infected corneal ulcer. These all responded to treatment with BTXA, topical antibiotics and/or lubrication, and eyelid surgery.

Conclusions Post-operative facial palsy may result in a significant ophthalmic workload.

Although a proportion of patients with a facial nerve palsy manage well with regular lubrication, additional help with eyelid closure, either in the way of BTXA-induced ptosis in the short term or definitive eyelid surgery in the long term, is often required. Eyelid surgery seems to be the mainstay of treatment, for both function and cosmesis, with many patients requiring a combination of procedures.
\end{abstract}

Key words Acoustic neuroma, Facial palsy, Botulinum toxin A, Eyelid surgery
Surgery for acoustic neuromas can be complicated by a post-operative lower motor neurone facial nerve palsy because of the intimate relationship between such tumours and the facial nerve.' The primary goal of the ophthalmologist in managing patients with facial nerve palsy is to protect the cornea from sight-threatening complications. Correction of eyelid malpositions, reducing epiphora and improving cosmesis are thus secondary goals, although they are of great importance to the patient. ${ }^{2,3}$ We have designed a clinical algorithm (Fig. 1) to aid in the ocular management of such post-operative patients so that sight-threatening complications may be reduced or avoided. The purpose of this study was to assess the usefulness and efficacy of this protocol and the treatments recommended in it Although initially designed for patients following acoustic neuroma excision, this flow diagram may of course be used in other situations where a facial palsy is encountered.

\section{Methods}

The hospital records of all consecutive patients undergoing primary surgery for excision of single acoustic neuromas during a 43 month period between January 1992 and August 1995 were studied. Patients with neuromas of other cranial nerves and those undergoing secondary surgery were excluded. The following details were recorded: age at surgery, sex, laterality, operation date, the absence or presence of a post-operative facial nerve palsy, its density and time to improvement, the management of any palsy, the presence or absence of any ocular or adnexal complications (including changes in vision). The House facial paralysis grading system was used, ${ }^{4}$ along with a simpler ophthalmic grading system to assess the density of palsy. If botulinum toxin A (BTXA) was required to induce a protective ptosis, then 10 units of toxin (Dysport) was injected through the central part of the upper eyelid skin crease with an insulin syringe.
S.A. Sadiq

R.N. Downes Department of Ophthalmology Queen's Medical Centre Nottingham NG7 2UH UK

Presented at the 3rd European Skull Base Congress, London, 9-11 April 1997 


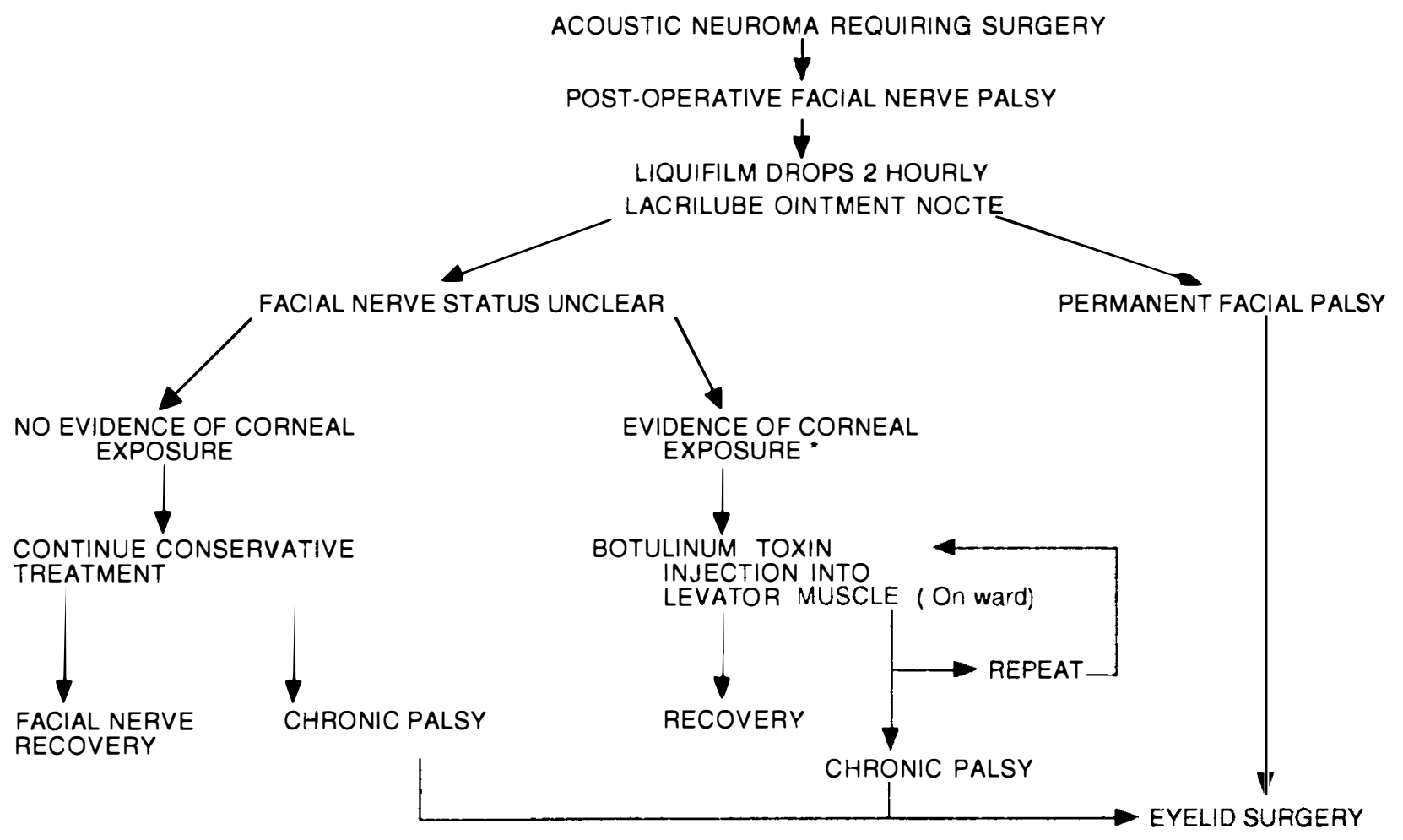

* Injected eye, corneal drying, comeal ulceration

Fig. 1. A clinical algorithm for the mamagement of facial ne're palsy following acoustic neuroma resection.

\section{Results}

Of all the patients undergoing primary acoustic neuroma excision during the above period, 48 sustained a chronic post-operative facial palsy (group A), and 8 sustained a temporary palsy (group B). The first 5 had been followed up prospectively by ourselves to ensure adequate understanding and working of the protocol by the neurosurgical staff.

\section{Group A}

The average age of the 48 patients in group A was 48.0 years (range 20-73 years) and the minimum follow-up period was 13 months (average 33.1 months, range 13-54 months). Twenty-four $(50 \%)$ were female and 24 were male. Thirty-one patients $(64.6 \%)$ had right-sided palsies, and $17(35.4 \%)$ had left-sided palsies.

Of the 48 patients, $39(81.3 \%)$ had a severe palsy from an ophthalmic viewpoint (i.e. incomplete eye closure, House grades IV-VI), 7 (14.6\%) had a moderate palsy (i.e. complete closure with effort, House grade III) and 2 $(4.2 \%)$ had a mild palsy (i.e. complete closure with minimal effort, House grade II).

During the follow-up period, $14(29.2 \%)$ were managed conservatively with regular lubrication only. Of these $14,7(50 \%)$ had a severe palsy, $5(35.7 \%)$ had a moderate palsy and $2(14.3 \%)$ had a mild palsy.

Nineteen patients required injection of BTXA to induce a protective ptosis either to treat exposure keratopathy or to promote healing of a corneal ulcer. Of these $19,18(94.7 \%)$ had a severe palsy and $1(5.3 \%)$ had a moderate palsy. Unfortunately, $8(42.1 \%)$ of the patients who had received BTXA suffered some amount of diplopia following resolution of the ptosis. Of these, in 4 the diplopia resolved completely (all had a mild superior or lateral rectus muscle underaction) and 4 were treated with either a Fresnel prism or ocular occlusion (all had a large or moderate superior rectus muscle underaction). None required squint surgery.

Twenty-four patients $(50 \%)$ required a total of 64 eyelid operations in one form or another, and the number and types of single procedures performed during the follow-up period are shown in Table 1. All these patients had severe palsies, except for 1 with a moderate palsy who required one procedure only. In only 7 patients $(29.2 \%)$ was a single procedure sufficient to prevent corneal exposure or improve patients' symptoms, with

Table 1. Types of eyelid surgery performed (total $=64)$

\begin{tabular}{lc}
\hline Procedure & No. \\
\hline Levator recession & 16 \\
Lateral tarsorraphy & 15 \\
Gold weight insertion & 10 \\
Medial canthoplasty & 6 \\
Lateral canthal sling & 5 \\
Gold weight removal & 5 \\
Wedge resection & 2 \\
Central tarsorraphy & 2 \\
Brow lift & 1 \\
Silicone sling insertion & 1 \\
Removal of silicone sling & 1 \\
\hline
\end{tabular}


Table 2. Number of surgical procedures required by patients

\begin{tabular}{cc}
\hline No. of procedures & No. of patients \\
\hline 1 & 7 \\
2 & 7 \\
3 & 3 \\
4 & 4 \\
5 & 2 \\
6 & 0 \\
7 & 0 \\
8 & 1 \\
\hline
\end{tabular}

the majority of patients having to undergo a variety of procedures. Table 2 shows the number of procedures undertaken during the follow-up period. Postoperatively, no patient had evidence of corneal exposure, and all felt that their cosmesis had improved.

Seven patients were referred with poor vision due to corneal epithelial defects or infected ulcers. The presenting vision ranged from perception of light to 6/9, and improved in all cases when more specialised treatment was instituted. The vision following treatment ranged from counting fingers (a patient with optic atrophy secondary to papilloedema) to $6 / 6$. Treatment involved BTXA injections in 6 cases (up to four injections in one patient) to induce a corneal protective ptosis. A central tarsorraphy was performed in one patient. This was accompanied by an increased frequency of lubrication (or antibiotics in the presence of infection). Definitive eyelid surgery was undertaken in 4 patients to abolish lagophthalmos once the epithelium had healed. Surgery consisted of levator recession in all 4 , lateral tarsorraphy in 3, medial canthoplasty in 2, a lateral canthal sling in 1 , and gold weight insertion in 1 case.

\section{Group B}

The average age of the 8 patients in group B was 52.3 years (range 25-68 years) and the minimum follow-up period was 12 months (average 31.3 months, range 12-54 months). Six $(75 \%)$ were female and 2 were male. Five patients $(62.5 \%)$ had right-sided palsies and $3(37.5 \%)$ had left-sided palsies. In the 8 patients, the palsy improved between 3 days and 6 months post-operatively (average 3.3 months). In the immediate post-operative period, 5 patients had mild palsies, 2 had a moderate palsy and 1 had a severe palsy. All patients were managed conservatively with regular lubrication, except one who required BTXA for signs of exposure keratitis (the only patient to have a severe palsy as well as trigeminal neuropathy). Unfortunately, this patient also developed residual diplopia due to moderate superior rectus underaction, which was managed with a Fresnel prism. Eyelid surgery was not required in this group.

\section{Discussion}

The eyelids provide a physical barrier to trauma and drying, and they inhibit adherence of organisms to the ocular surface. Tears provide mechanical lubrication to wash away organisms, and they also contain antimicrobial substances. If lid closure is defective, as after a facial palsy, then the tear film is unable to provide coverage and the epithelium becomes susceptible to drying. In addition to poor eyelid closure following facial palsy, patients who have undergone acoustic neuroma resection may also have decreased corneal sensation preor post-operatively, as well as decreased vasomotor tone to the lacrimal gland. These further factors also contribute towards an unprotected and anaesthetic dry eye. It is such an eye that is at high risk of ulceration, infection and scarring, with consequent reduction in vision.

Although it is the primary function of the eyelids to protect the cornea, a secondary eyelid function is to participate with other muscles in facial expression, and it is a weakness in the latter function which seems to be of most concern to the patients themselves. ${ }^{3}$ A facial palsy can prevent patients from resuming their former social activities $^{2}$ and employment, as well as adding to psychological problems.' Once corneal protection has been achieved by either lubrication, BTXA or initial eyelid surgery, the aims of further eyelid surgery are to restore function to the eyelids and upper face. We, along with other authors, " believe this reanimation is best managed in a staged approach by passive or dynamic means. Any procedure used in facial palsy should (1) provide adequate corneal protection, (2) be cosmetically acceptable, (3) avoid visual disturbance, (4) create facial symmetry, (5) be reversible in the event of spontaneous palsy improvement, and (6) be technically easy. ${ }^{7}$

Assessment of the density of a facial palsy is usually made by neurosurgeons and otorhinolaryngologists using the House facial paralysis grading system.' Although the House grading system has been used for the purpose of this study, we prefer to grade a facial nerve palsy clinically as mild, moderate or severe depending on the amount of eyelid closure achieved as mentioned in Results. The other observations to be made, especially when assessing with a view to definitive eyelid surgery, include the vertical palpebral aperture, the presence of any brow droop, upper eyelid retraction, lower eyelid laxity, scleral show or risk of corneal exposure.

The first part of our algorithm requires regular ocular lubrication with drops in the daytime and ointment at nightime. Just over a third of our patients required lubrication only which indicates that ophthalmic referral is probably not indicated (or practicable) in all cases of facial palsy. Lubrication may be sufficient to provide ocular comfort and prevent corneal exposure in patients with mild or moderate palsies, but is rarely adequate in those with severe palsies. Eyelid surgery should be performed early in the presence of corneal exposure or epithelial defects, or if the patient is known to have a permanent palsy (e.g. the facial nerve had to be sacrificed during acoustic neuroma resection). If the prognosis of the palsy is unknown (e.g. the facial nerve was anatomically and/or electrophysiologically preserved during acoustic neuroma resection), then it would be prudent to wait for 12 months to allow for any 
spontaneous improvement before embarking on definitive eyelid surgery. Our results show that normal facial function does not seem to occur if more than 6 months have elapsed since acoustic neuroma surgery.

BTXA injection does provide a good means of providing corneal coverage by paralysing the levator palpebrae muscle and so inducing a ptosis, especially when a patient is unable to undergo eyelid surgery (e.g. whilst in intensive care). BXTA is minimally invasive and can be performed without any anaesthesia at all. It allows for easy ocular examination and instillation of any topical treatment required, whilst at the same time it does not cause any eyelid scarring. A $45 \%$ rate of complications following BTXA in the form of rectus muscle underaction and resulting diplopia (although half resolve quickly) is cause for concern, but is less than the rate when BTXA is given along the roof of the orbit. ${ }^{8}$ Although there were no persistent cases of diplopia in the original series, squint surgery has been required in subsequent patients receiving BTXA along the roof of the orbit." We feel that our lower rate of diplopia occurrence may be due to the lower site of injection, i.e. through the skin crease rather than below the superior orbital margin, and this may allow less spread of toxin to the adjacent recti. We are at present investigating alternative injection methods and sites for BTXA in order to reduce the associated complications.

Although the advent of BTXA has meant that we have no longer had to resort to central tarsorraphies, a lateral tarsorraphy is probably still the commonest static eyelid procedure performed for patients with a facial palsy by many surgeons. We rarely perform this procedure on its own as it is functionally and cosmetically poor, and can render the eyelids immobile in an open position. ${ }^{6}$ It may be useful as a temporary measure, especially when combined with other eyelid procedures. A lateral tarsorraphy may also restrict the visual field, and inclusion cysts may occur along the line of re-opening. Medial and lateral canthoplasty procedures tighten the lower eyelid and oppose it to the globe by shortening or redirecting the pull of the canthal tendons. ${ }^{10}$

Canthoplasty also does not fixate the lids in an open position as may a lateral tarsorraphy.

Unopposed action of the levator palpebrae muscle may lead to upper eyelid retraction and a staring appearance. This can be dealt with by way of a levator recession. This is easily performed via a posterior approach, and the amount of recession can be titrated to the amount of eyelid retraction present. The procedure is also repeatable. Reversal is possible if overcorrection occurs. Levator recession is often performed in combination with static eyelid surgery, such as canthoplasty or tarsorraphy. In patients with coexisting trigeminal neuropathy ( 16 of 56 of our patients), the eye is at even greater risk, and in such circumstances we do aim for an overcorrection so that the eyelid is ptotic.

Passive reanimation of the upper eyelid can be performed with gold weight loading, which allows gravity-aided passive eyelid closure and blink whilst erect. As it is gravity dependent, it may not cause closure when supine, although a better mass effect is obtained by placing the weight inferiorly. ${ }^{6}$ Symptoms of nocturnal lagophthalmos may be alleviated by eyelid taping just before sleep. The weight can also help to reduce eyelid retraction, ${ }^{11}$ and we find it useful if levator surgery is not sufficient to prevent corneal exposure. It is easily performed and easily reversed and has many advocates. ${ }^{11-14}$ However, we have experienced a high rate of removal of gold weights for reasons of poor cosmesis beneath the thin eyelid skin, persisting overlying erythema, extrusion and overcorrection with resulting ptosis. These same features have also been reported by others. ${ }^{15}$

Dynamic eyelid reanimation is possible with the use of palpebral springs and silicone slings. They have a closing effect regardless of gravity, and provide a faster blink with better and more frequent corneal coverage. ${ }^{6}$ We have tended not to use these methods as these devices have been reported to have a high level of extrusion, and may require subsequent revision. ${ }^{6,7}$ Various ancillary procedures such as a direct brow lift may be useful in the later stages to help improve cosmesis and increase the visual field superiorly if there is significant brow droop overhanging the visual axis. This should be performed in the presence of good corneal lubrication as the procedure may worsen an individual's ability to close the eyes. ${ }^{6}$

In conclusion, although a proportion of patients with a facial nerve palsy manage well with regular lubrication, most require some additional help with eyelid closure, either in the way of BTXA-induced ptosis in the short term or definitive eyelid surgery in the long term. The former seems to afford good corneal protection, especially in emergency situations where there is an epithelial defect or an infected corneal ulcer. The method and site of injection of BTXA do require refinement to reduce the rate of complications, even though some of these are temporary. Eyelid surgery seems to be the mainstay of treatment, for both function and cosmesis. There are many procedures that may be undertaken - a fact which indicates that no one procedure is the ideal. Indeed many patients seem to require a combination of procedures, which is borne out by other authors also. ${ }^{16}$

\section{References}

1. Wright A, Bradford R. Management of acoustic neuroma. BMJ 1995;311:1141-4.

2. Kane N, Kazanas S, Maw A, et al. Functional outcome in patients after excision of extracanalicular acoustic neuromas using the suboccipital approach. Ann R Coll Surg Eng 1995;77:210-6.

3. Kartush J, Lundy L. Facial nerve outcome in acoustic neuroma surgery [review]. Otolaryngol Clin North Am 1992;25:623-47.

4. House J. Facial nerve grading systems. Laryngoscope 1983;93:1056-69.

5. Jorgensen B, Pedersen C. Acoustic neuroma: follow-up of 78 patients. Clin Otolaryngol 1994;19:478-84.

6. Seiff S, Chang J. Management of ophthalmic complications of facial nerve palsy. Otolaryngol Clin North Am 1992;25:669-90. 
7. Sobol S, Alward P. Early gold weight lid implant for rehabilitation of faulty eyelid closure with facial paralysis: an alternative to tarsorraphy. Head Neck 1990;[March/ April]:149-53.

8. Adams G, Kirkness C, Lee J. Botulinum toxin A induced protective ptosis. Eye 1987;1:603-8.

9. Heyworth P, Lee J. Persisting hypotropias following protective ptosis induced by botulinum neurotoxin. Eye 1994;8:511-5.

10. Levine R. Reanimation of paralysed eyelids. Facial Plast Surg 1992;8:121-6.

11. O'Connell J, Robin P. Eyelid gold weights in the management of facial palsy. J Laryngol Otol 1991;105:471-4.
12. Seiff R, Sullivan J, Freeman N, Ahn J. Pretarsal fixation of gold weights in facial nerve palsy. Ophthalmic Plast Reconstr Surg 1989;5:104-9.

13. Kartush J, Linstrom C, McCann P, Graham M. Early gold weight eyelid implantation for facial paralysis. Otolaryngol Head Neck Surg 1990;103:1016-23.

14. Gilbard S, Daspit C. Reanimation of the paretic eyelid using gold weight implantation. Ophthalmic Plast Reconstr Surg 1991;7:93-103.

15. Pickford M, Scamp T, Harrison D. Morbidity after gold weight insertion into the upper eyelid in facial palsy. $\mathrm{Br} \mathrm{J}$ Plast Surg 1992;45:460-4.

16. Leatherbarrow B, Collin J. Eyelid surgery in facial palsy. Eye 1991;5:585-90. 